\title{
A Hybrid Overlay Network for Video-on-Demand
}

\author{
Ming Zhou and Jiangchuan Liu \\ School of Computing Science \\ Simon Fraser University, Burnaby, British Columbia, Canada \\ \{jmzhou,jcliu\}@cs.sfu.ca
}

\begin{abstract}
On-demand video streaming through overlay networks has received much attention recently. While a tree topology is often advocated in such systems, it suffers from discontinuous playback under the highly dynamic Internet environment with frequent node joins and leaves. On the other hand, gossip protocols using random message dissemination, though robust, fail to meet the real-time demands for streaming applications. In this paper, we propose HON, a Hybrid Overlay Network protocol, which combines the best features of these two approaches for ondemand streaming: low delay with a regular tree topology, and robust delivery with random switching among multiple paths, thus making effective use of the available bandwidth in the network. We design an adaptive tree construction and gossip management algorithm for HON, and evaluate its performance under various settings. The results demonstrate that $\mathrm{HON}$ is quite robust in the presence of local and global bandwidth fluctuations. As compared to pure tree-based overlay VOD system, it achieves much lower and stable segment missing rates, even under highly dynamic network conditions.
\end{abstract}

Keywords - Overlay network, Video-on-demand, Gossip protocol, Peer-to-peer communication

\section{INTRODUCTION}

With the exponential expansion of Internet resources and users, Video-on-Demand(VoD) has become one of the most attractive services over the Internet. Nevertheless, for $\mathrm{VoD}$ to hundreds or thousands of clients, the traditional clientserver model generally fails, due not only to the network bandwidth constraints but also to the unbearable server stress. There have been many proposals on providing scalable on-demand streaming through multicast or proxy caching; yet the deployment of IP multicast and dedicated proxies remains limited nowadays. Recently, application-layer communication offers a promising alternative solution, which builds an overlay network out of unicast tunnels across cooperative peers with certain buffering capabilities, called overlay nodes [1]-[3]. Each overlay node acts as an application-layer proxy, and multicast can also be achieved through data relaying among them. Several pioneering works have demonstrated that scalable on demand video streaming in a peer-to-peer overlay network is viable [8]-

\footnotetext{
${ }^{1}$ This work is partially supported by a Canadian NSERC Discovery Grant and SFU President's Research Grant.
}

[13]. As an example, in oStream [8], each node in the system cache the most recent content of the video it receives; existing nodes in the system can forward the video data to a new client as long as it has enough outbound bandwidth and the data segments are still in its buffer. The ordered forwarding and receiving relations lead to a tree structure, which is natural and efficient for multicasting, and is also often advocated in other existing systems.

There are, however, several critical problems for a treebased overlay: first, each node relies on a single path from source to retrieve data; bandwidth fluctuations at this specific path may results in highly unstable video quality; second, only paths in the tree are used to deliver data; paths close to the root thus often become bottlenecks, while all other potential paths are untouched, leading to poor resource utilization; and third, the leave of internal nodes, especially those close to the root, may cause buffer underflow at a large population of downstream nodes; such situations are not uncommon as overlay nodes can join or leave at will.

Opposite to a tree-based protocol, gossip protocols enable random data dissemination without support from a regular overlay structure [5]-[7]. In a typical gossip process, a node randomly selects $K$ target nodes to deliver recently available data segments, and meanwhile, receives segments pushed from other $K$ nodes. It is known that, for gossip, the loss probability is exponentially small in a network of $n$ nodes with $K$ equal to $c+\log (n)$ where $c$ is a constant [6]. As such, it is quite robust. Nevertheless, it is not straightforward to apply gossip in on-demand streaming, for it often fails to achieve a timely delivery. Furthermore, the push-based gossip could cause excessive duplications, which is particularly severe for high-bandwidth videos.

In this paper, we present $\mathrm{HON}$, a Hybrid Overlay Network protocol for on-demand media streaming. HON constructs and maintains two overlays, namely, a tree overlay and a gossip overlay, which collectively deliver video contents to clients of asynchronous demands. We design intelligent and efficient overlay construction and data scheduling algorithms to facilitate streaming in this hybrid system. In particular, most data segments are delivered through the gossip overlay; only if a node fails to receive a data segment till certain deadline, will it resort to the tree overlay to fetch the segment from its parent. As such, each node can accommodate far more children than in the pure-treestructured scheme. More importantly, the random dissemination in gossip enables segment delivering from multiple sources, which not only exploits the available 
which not only exploits the available bandwidth from all the potential network paths, but also enhances robustness in the presence of bandwidth oscillations or malfunctions of internal tree nodes. We also substitute the push mechanism in both the gossip and the tree-structured streaming by a pull process, which greatly eliminates the redundancy due to random disseminations.

We evaluate the performance of $\mathrm{HON}$ under various network configurations. The results demonstrate that it is highly robust when facing local and global bandwidth fluctuations. As compared a pure tree-based overlay VoD system, it achieves much lower and stable segment missing rates, even under highly dynamic network environments.

The rest of the paper is organized as follows. The system model of HON is presented in Section II, with its operations detailed in Section III. The performance of HON is evaluated in Section IV. Finally, Section V concludes the paper and offers some future research directions.

\section{THE HON MODEL}

Fig. 1 depicts a generic HON model, which maintains two overlay structures that collectively deliver video data to receivers of asynchronous demands. One is a random graph for gossip (solid lines), and the other is a tree (dashed line), which is rooted at the source node of the videos (node $S$ ).

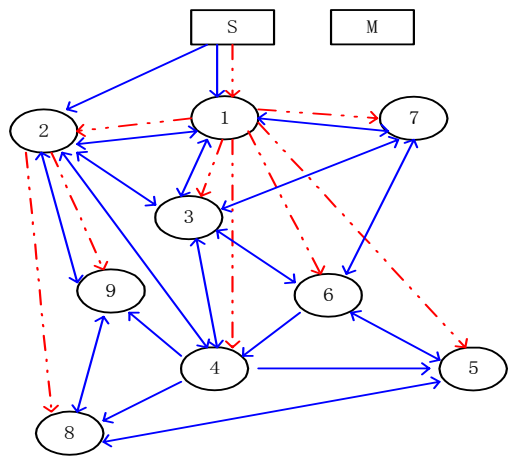

Figure1. The HON Model. S: Source node; M: Managing node.

A HON node should participate in both overlays, e.g., node 3 is a child of node 1 in the tree overlay, and meanwhile, is a gossip source or target of nodes $1,2,4,6,7$. When a node joins the system, it begins to fetch data segments from its gossip sources. Specifically, whenever a gossip source obtains a new data segment, it sends a data offer to its gossip targets, and that target node will then decide whether to accept the offer. If so, it will send a pull request to the gossip source to fetch the segment, thus avoiding duplications. Note that gossip does not guarantee timely delivery. Hence, when a data segment to be played out is not available in the buffer till a certain threshold, the node should contact its parent to retrieve the segment. Yet, this happens infrequently as revealed in our experiments, implying that the tree overlay mainly serves as a rescuing mechanism.

The two overlays in HON are constructed and maintained by a managing node, which is a dedicated node persisting during in the lifetime of the streaming. An example is the source node that provides the video content. As will be shown later, the operations in the managing node are indeed simple, which incur low computation as well communication overheads. In addition, to facilitate the 2-overlay delivering, the buffer of each node is divided into two parts: a backward and a forward buffer. The former caches the data segments that have been played out at the node, and might be used to serve its children in the tree overlay. The later, however, is used for the gossip process, which stores data segments that are delivered in advance.

\section{HON OPERATIONS}

In this section, we discuss the HON operations in detail. As said, the HON managing node is responsible for generating and maintaining the tree structure as well as the partner relationships for gossiping. It keeps tracking the following fields for all the nodes: $I D_{i}$, the identification of node $i$, e.g., IP address; $p_{i}$, the starting play-out offset at node $i$; $b_{i}^{+}$, the forward buffer size at node $i$ (in units of playback time); $b_{i}^{-}$, the backward buffer size at node $i$ (in units of playback time); $B_{i}$, the available bandwidth at node $i$ for its children in the tree; $t_{i}$, the time when a subscription request arrives at the managing node; inList, the list of neighboring nodes from which node $i$ receives gossip information; and outList, the list of neighboring nodes to which node $i$ sends gossip information

For simplicity, we assume that a video stream consists of $M$ segments, indexed from 1 to $M$, each with a unit playback time. Let $t$ be the current time (in units); $\left(t-t_{i}+p_{i}\right)$ is thus the segment sequence number be played at node $i$.

Each node is assigned a parent in the tree. The set of potential parents for node $i$ include any nodes $j$ that satisfies:

$$
\text { ( Condition 1) }\left\{\begin{array}{l}
p_{j}-t_{j}>p_{i}-t_{i} \\
p_{j}-t_{j}-b_{j}^{-}<p_{i}-t_{i} \\
B_{j} \geq B_{\text {video }}
\end{array}\right.
$$

which basically implies that the constraints of buffer size and playback time have to be met. In case more than one candidate that satisfies Condition 1, we select the one with the highest playback segment sequence number so far. Intuitively, such a node is closer to the root, and hence a tree with lower depth will be constructed, yielding lower delay.

We now consider the criteria for choosing the gossip partners at each node. Due to the limit of buffer size and the asynchronism, node $i$ generally has a dynamic group of gossip targets. This is illustrated in Fig. 2, where $q_{i}$ represents $t-t_{i}+p_{i}$, i.e., the current playout segment sequence number at node $i$ : 


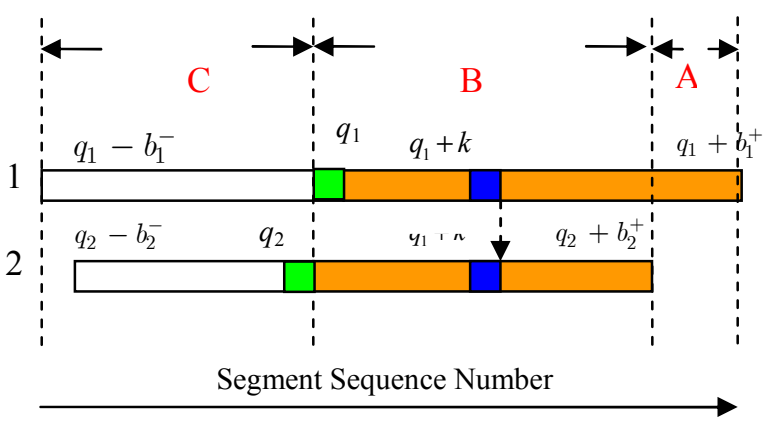

$\begin{array}{lll}\text { Forward Buffer } & \square \quad \text { Segment played out } \\ \text { Backward Buffer } & \square \quad \text { Segment to be gossiped }\end{array}$

Figure 2. An illustration for the $k$-group condition.

In Fig. 2, a segment available in node 1's forward buffer can not necessarily be gossiped to node 2 . In particular, if it falls into area $\mathrm{C}$, it is already played out at node 2; if it falls into area A, it is outside of node 2's forward buffer. Thus, only segment in area B should be gossiped. In other words, whether two nodes can form a partnership for gossip is related to the offset value $k$ of the segment to be forwarded. We refer to the set of nodes that can be partners of node $i$ to gossip a segment with offset $k$ as a $k$-group of node $i$. Clearly, a node $j$ in the $k$-group should satisfy the following conditions:

$$
\text { ( Condition2 ) }\left\{\begin{array}{l}
q_{i}+k \geq \max \left(q_{i}, q_{j}\right) \\
q_{i}+k \leq \min \left(q_{i}+b_{i}^{+}, q_{j}+b_{j}^{+}\right)
\end{array}\right.
$$

where $q_{i}=t-t_{i}+p_{i}$ and $q_{j}=t-t_{j}+p_{j}$.

When a segment is received at node $i$ with offset $k$ to its playout segment, the segment can be gossiped in node $i$ 's $k$ group. Ideally, for all the possible values of $k$, the corresponding segment should be gossiped. To make the operation simpler and yet robust, in our implementation, a segment will be gossiped at most $d$ rounds, each round with a randomly selected and unique value of $k$.

Given the above criteria for parent and gossip partner selection, we now detail the operations in the systems.

\section{A. Node Subscription}

When a new node $(i)$ joins the system, the following operations are to be performed:

\section{For node $i$ :}

Send the managing node a Request message of form $\left(I D_{i}, p_{i}, b_{i}^{+}, b_{i}^{-}, B_{i}\right)$.

\section{For the managing node:}

1. Generate a record for node $i$, including all fields in the above request, plus field $t_{i}$, the time when the request is received. The record is then stored into a control record list sorted in descending order of $\left(p_{i}-t_{i}\right)$;

2. Search from the front of the control block list, find the first control record that satisfies Condition 1, and update its Parent and children fields according to the criteria we have discussed. If no parent is found, the managing node will serve as the parent;

3. Randomly select $k$-groups for node $i$ with $k$ in between 1 and $b_{i}^{+}$, and add the group members to a set $S$. This step continues until $|S| \geq 2 K$ where $K$ is a predefined threshold, or all the $k$-groups have been enumerated;

4. Divide set $S$ into two equal halves: inList and outList. The corresponding fields in the related control record are updated to build the gossip partnership;

5.Reply node $i$ with a Join message of form (parent, inList, outList).

Finally, node $i$ should contact its parent and neighbors assigned by the managing node to establish parent-children relationship and the gossip partnership.

\section{B. Node Unsubscription}

When node $i$ gracefully departs from the system, the following operations are to be performed:

\section{For node $i$ :}

1. Send a Departure message to its Parent, Children, as well as nodes in its inList and outList, such that the corresponding fields can be updated;

2. Send an Unsubscribe message to the managing node, including the gossip neighborhood changing information.

\section{For managing node:}

1. Update the inList and outList fields of the related nodes, and search for new parent for the children of node $i$ using the same method as in node subscription;

2. Send messages to node $i$ 's children to assign new parents.

\section{Segments Dissemination}

Given the parent, inList and outList assigned by the managing node, a newly joined node can then establish the relations with other nodes in the tree structure and the gossip graph, and start fetching and forwarding data segments.

When node $i$ receives a data segment in its forward buffer with offset $k$ to its play-out segment, it first calculates its k-group in its outList, and sends a data availability information about the received data segment to the nodes in its $k$-group. Those expecting the segment should send a request, and the segment is then delivered by node $i$. As men- 
tioned before, $d$ rounds of gossip with different values of $k$ are to be executed for each segment to balance overhead and robustness.

When a segment is not available at a node for playout within a threshold (the round-trip time between the node and its parent plus the segment transmission time), the node should contact its parent to fetch the segment.

Note that the availability information and fetch request are quite small, which can also be piggybacked in data segments. In addition, since a node can start receiving and playing back from any data segment, VCR functionalities such as forward and backward can be easily incorporated by initiating a new subscription request. Given the gossip nature and the dynamic tree construction, a node can also stop receiving data and pause the playback at will without affecting downstream nodes.

\section{Failure Detection and Recovery}

When a node is found dead by its neighbor, e.g., due to no response for a long time, the neighbor should report this to the managing node. The managing node, upon receiving more than one report for the same node, informs all the parent, children, inList and outList of the dead node, and assign new parents and gossip partners using similar methods as in node subscription.

\section{Performance Evaluation}

We evaluate the performance of $\mathrm{HON}$ under various network settings, and also compare it with other overlay ondemand streaming systems, in particular, oStream, a pure tree-based system. In our evaluation, the underlying network topology is generated using the GT-ITM package [14]. The network consists of 3 transit domains, each with 4 transit nodes, and a transit node is then connected to 4 stub domains, each with 4 stub nodes. The total number of nodes is thus 200. The initial bandwidth assigned to the links is as follows: each link between two stub nodes can support 6 concurrent streams, each link between a stub node and a transit node can support $4 \times 6=24$ concurrent streams, and each link between two transit nodes can support $6 \times 6=36$ concurrent streams.

We assume routing between two nodes in the network follows the shortest path algorithm. The source node has 10 videos for streaming. The length of each video is 2 hours, and that of each segment (or time unit) is 1 second. The client nodes participating in the system follow a Poisson arrival, each being randomly attached to a network node and requesting the video from a random starting point from 0 to 2 hours. The buffer at each client node can accommodate 12 -minute video, or $10 \%$ of a video stream, and is equally split into forward and backward buffers. Each nodes gossip 2 rounds, with 6 in-neighbors and 6 out-neighbors for gossip process. Regarding oStream for comparison, similar network and buffer settings are adopted.
Since playback continuity is critical for streaming applications, we adopt the Segment Missing Rate (SMR) as the major metric in this evaluation. A data segment is considered missing if it is not available at a node till the playout time, and the SMR for the whole system is the average ratio of the missed segments at all the participating nodes during the simulation time. As such, it reflects two important aspects of the system performance, namely, delay and capacity.

Due to space limit, we now present some typical results under dynamic network environments. In a stable environment, HON achieves even better performance by effectively exploring the available bandwidth in the network.

\section{A. Effect of Local Bandwidth Fluctuation}

In the first set experiments, we examine the impact of local bandwidth fluctuations in a dynamic network. We randomly inject traffic to the network links such that the available bandwidth at each link various over time, yet the total available bandwidth of the network remains constant, which is 0.8 of the base (with no cross traffic).

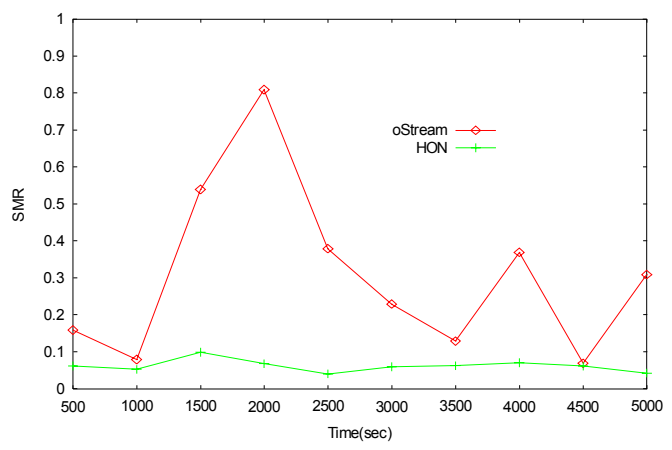

Figure 3. Segment loss rate (SMR) over time.

Fig. 3 shows the segment loss rates (SMRs) for HON and oStream over time. It can be seen that the loss rate of HON is not only lower than oStream, but also quite stable, which is generally around 0.07 to 0.1 . From a video decoding point of view, such a loss rate is reasonably low and can be effectively masked by interleaving or error-concealment techniques. On the other hand, the loss rate of oStream greatly fluctuates over time, and the peak value can be as high as 0.8 , resulting in poor video quality. This is because oStream relies on a specific tree structure for streaming, and the bandwidth reduction at an internal link of the tree, especially those close to the root, could result in a lot of segment losses in downstream nodes. HON employs random message dissemination that adaptively picks up high-quality delivering paths and is thus more robust in the presence of bandwidth fluctuations. In other words, gossip can effectively complement a tree structure in a dynamic network, and yet, with the assistance from the tree structure, no excessive delay is introduced as suggested by the experiment results.

\section{B. Effect of Global Bandwidth Fluctuation}


It is known that not only the available bandwidth of local links dynamically changes, but also the overall available bandwidth of a network changes over time on an hour or daily basis, e.g., working and sleeping hours, and working days and weekends, etc. Hence, in the second set of experiments, we compare the performance of HON and oStream under different global network bandwidths. Their segment loss rates are depicted in Fig. 4, where the overall available bandwidth of the network is gradually reduced from $100 \%$ to $50 \%$ of the base.

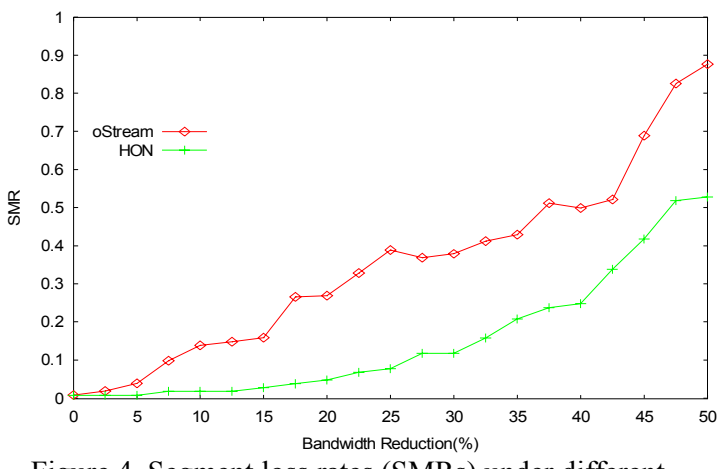

Figure 4. Segment loss rates (SMRs) under different overall network bandwidths.

Not surprisingly, for both HON and oStream, SMR increases with decreasing overall bandwidth. However, the increasing rate for $\mathrm{HON}$ is generally lower than that of oStream, especially when the reduction is less than $25 \%$. As an example, for a reduction of $25 \%$, the SMR of oStream has reached 0.4 , or $40 \%$ of the segments are lost or missed the playback deadline, yet the SMR of HON remains lower than 0.1 . This is because oStream uses the available bandwidth at a subset of the links only (i.e., only those in the tree), while HON makes effective use of the available bandwidth across all paths. In addition, once a segment is lost at a high level node in oStream, it will be lost at all downstream nodes. This is, however, not the case in HON for each node has multiple possible data sources. Finally, note that the SMR for oStream is not monotonically decreasing, which again reflects its instability, as observed in Fig. 3.

\section{CONCLUSION AND FUTURE WORK}

In this paper, we have presented HON, a hybrid overlay network protocol for on-demand media streaming. HON has combined the best features of tree structure and random message dissemination: low delay with a regular tree topology, and robust delivery with random switching among multiple paths, which make effective use of the available bandwidth in the network. The performance of HON has been extensively evaluated under various network configurations. The results demonstrated that it is highly robust in the presence of local and global bandwidth fluctuations. As compared pure tree-based overlay VOD system, HON achieves much lower and stable segment missing rates, even under highly dynamic network environments.
We are currently building an Internet-based prototype for HON, and preparing for experiments over the PlanetLab. Possible further research avenues include designing a fully distributed protocol, optimizing the delivering paths, and incorporating network coding techniques. We expect more issues to be identified and solved in this prototyping process.

\section{REFERENCES}

[1] Y. Chu, S. Rao, and H. Zhang, "A case for end system multicast," in Proc. ACM SIGMETRICS, Santa Clara, CA, 2000.

[2] D. Andersen, H. Balakrishnan, M. Kaashoek, and R. Morris, "Resillient overlay network," in Proc. ACM SOSP, Banff, Canada, 2001

[3] D. Xu, M. Hefeeda, S. Hambrusch, and B. Bhargava, "On peerto-peer media streaming," in Proc. ICDCS'02, Wien, Austria, Jul. 2002

[4] S. Banerjee, B. Bhattacharjee, and A. Srinivasan, "Resilient multicast using overlays," in Proc. ACM SIGMETRICS'03, San Diego, CA, USA, Jun. 2003.

[5] P. Eugster, R. Guerraoui, A.-M.,Kermarrec, and L. Massoulie, "From epidemics to distributed computing," to appear in IEEE Computer Magazine, 2004.

[6] A.J. Ganesh, A.-M.Kermarrec, and L. Massoulie, "Peer-to-peer memebership management for gossip-based protocols," IEEE Trans. on Computer,52(2), Feb. 2003.

[7] X. Zhang, J. Liu, B. Li, and T. -S. P. Yum, "CoolStreaming/DONet: a data-driven overlay network for peer-to-peer live media streaming," in Proc. IEEE INFOCOM'05, Mar. 2005.

[8] Y. Cui, B. Li, and K. Nahrstedt, "oStream: asynchronous streaming multicast," IEEE J. Select. Areas in Comm,Jan. 2004

[9] Y. Guo, K. Suh, J. Kurose, and D. Towsley, "P2Cast: peer-topeer patching scheme for VoD service," in Proc. $W W W^{\prime} 03$, Budapest, Hungary, May 2003.

[10] T. Do, K. A. Hua, and M. Tantaoui, "P2VoD: providing fault tolerant video-on-demand streaming in peer-to-peer environment," in Proc. ICC'04, Paris, France, Jun. 2004.

[11] Y. Cui and K. Nahrstedt, "Layered peer-to-peer streaming," in Proc. NOSSDAV'03, Monterey, CA, USA, Jun., 2003.

[12] Y. Guo, K. Suh, J. Kurose, and D. Towsley, "A peer-to-peer on-demand streaming service and its performance evaluation," in Proc. ICME 2003, Baltimore, MD, July 2003.

[13] M. Hefeeda and B. Bhargava, "On-demand media streaming over the Internet," In Proc. FTDCS'03, San Juan, Puerto Rico, May,2003.

[14] E. Zegura, K. Calvert, and S. Bhattacharjee, "How to model an internetwork," in Proc. IEEE INFOCOMM, San Francisco, CA,USA, Mar.,1996. 